International Journal of Applied Mathematics

Volume 29 No. $2 \quad 2016,189-201$

ISSN: 1311-1728 (printed version); ISSN: 1314-8060 (on-line version)

doi: http://dx.doi.org/10.12732/ijam.v29i2.3

\title{
FRACTIONAL CALCULUS OPERATORS OF GENERALIZED CONFLUENT HYPERGEOMETRIC FUNCTION
}

\author{
Jai Prakash Patel ${ }^{1}$, Neelam Pandey ${ }^{2}$ \\ ${ }^{1,2}$ Department of Mathematics \\ Govt. Model Science College \\ Rewa, 486003, INDIA
}

\begin{abstract}
In the present paper, the authors establish some fractional integral and fractional derivative formulas involving a generalized confluent type hypergeometric function introduced by Parmer [6].
\end{abstract}

AMS Subject Classification: 26A33, 33B15, 33C05, 33C20, 44A10, 44A20, 33B99, 33C99, 33D90

Key Words: extended Beta function, fractional integral and derivative operators, extended generalized hypergeometric functions

\section{Introduction}

The special functions play important role in mathematics and its diverse fields. In particular, the hypergeometric function is involved in solving numerous problem of mathematical physics, engineering and applied mathematics (see Ozergin [5], Samko et al. [11], Kiryakova [3], [4], Kilbas et al. [2], Prajapati and Kachhia [8], Kachhia and Prajapati [1], etc.). This inspires the study of several generalizations of the hypergeometric functions. Before starting and proving our results, we present some notations, basic definitions and preliminary results useful for the further discussions.

Received: December 28, $2015 \quad$ (c) 2016 Academic Publications

${ }^{\S}$ Correspondence author 
We also recall the Pochhammer symbol $(\lambda)_{n}$ defined (for $\lambda \in \mathbb{C}$ ) as (Rainville $[9])$

$$
(\lambda)_{n}=\left\{\begin{array}{l}
1, \quad(n=0), \\
\lambda(\lambda+1) \ldots(\lambda+n-1) \quad(n \in \mathbb{N}) \\
\frac{\Gamma(\lambda+n)}{\Gamma(\lambda)}, \quad\left(\lambda \in \mathbb{C} / \mathbb{Z}_{0}^{-}\right),
\end{array}\right.
$$

where $\mathbb{Z}_{0}^{-}$denotes the set of non positive integers.

The classical Beta function is defined by (Rainville [9])

$$
B(x, y)=\int_{0}^{1} t^{x-1}(1-t)^{y-1} d t ; \quad \operatorname{Re}(x)>0, \operatorname{Re}(y)>0
$$

Recently, a generalization of the beta function has been given by Parmar [6] as follows:

$$
B_{\gamma}^{(\alpha, \beta ; \mu)}(x, y)=\int_{0}^{1} t^{x-1}(1-t)^{y-1}{ }_{1} F_{1}\left(\alpha ; \beta ; \frac{-\gamma}{t^{\mu}(1-t)^{\mu}}\right) d t
$$

where $\mathfrak{R}(p)>0, \mathfrak{R}(x)>0, \mathfrak{R}(y)>0, \mathfrak{R}(\alpha)>0, \mathfrak{R}(\beta)>0$ and $\mathfrak{R}(\mu)>0$.

It is interesting to observe that for $p=0$, the generalized Beta function (1.3) reduces to the classical gamma function (1.2).

The confluent hypergeometric function is defined by (Rainville [9])

$$
{ }_{p} F_{q}\left[\begin{array}{c}
a_{1}, \ldots, a_{p} \\
b_{1}, \ldots, b_{q}
\end{array} ; z\right]=\sum_{n=0}^{\infty} \frac{\left(a_{1}\right)_{n}, \ldots,\left(a_{p}\right)_{n}}{\left(b_{1}\right)_{n}, \ldots,\left(b_{q}\right)_{n}} \frac{z^{n}}{n !}
$$

with $p \leq q$ or $p=q+1$ and $|z|<1$.

The generalized confluent hypergeometric function ${ }_{p} F_{q}^{(\alpha, \beta ; \gamma)}\left[\begin{array}{c}a_{1}, \ldots, a_{p} ; z ; \gamma \\ b_{1}, \ldots, b_{q}\end{array}\right]$ can be defined as (see Parmar [6])

$$
{ }_{p} F_{q}^{(\alpha, \beta ; \gamma)}\left[\begin{array}{c}
a_{1}, \ldots, a_{p} \\
b_{1}, \ldots, b_{q}
\end{array} ; z ; \gamma\right]:=\sum_{n=0}^{\infty} \Theta(n / p, q) \frac{z^{n}}{n !}
$$


where $\gamma \geq 0$ and the coefficient term $\Theta(n / p, q)$ is expressed by

$$
\Theta(n / p, q)=\left\{\begin{array}{l}
\left(a_{1}\right)_{n} \prod_{j=1}^{q} \frac{\mathcal{B}_{\gamma}^{(\alpha, \beta ; \kappa)}\left(a_{j+1}+n, b_{j}-a_{j+1}\right)}{\mathcal{B}\left(a_{j+1}, b_{j}-a_{j+1}\right)}, \\
\quad\left(p=q+1 ; \Re\left(b_{j}\right)>\Re\left(a_{j+1}\right)>0 ;|z|<1\right), \\
\prod_{j=1}^{q} \frac{\mathcal{B}_{\gamma}^{(\alpha, \beta ; \kappa)}\left(a_{j}+n, b_{j}-a_{j}\right)}{\mathcal{B}\left(a_{j}, b_{j}-a_{j}\right)}, \\
\quad\left(p=q ; \Re\left(b_{j}\right)>\Re\left(a_{j}\right)>0 ; z \in \mathbb{C}\right), \\
{[4 p t] \prod_{i=1}^{r} \frac{1}{\left(b_{i}\right)_{n}} \prod_{j=1}^{p} \frac{\mathcal{B}_{\gamma}^{(\alpha, \beta ; \kappa)}\left(a_{j}+n, b_{r+j}-a_{j}\right)}{\mathcal{B}\left(a_{j}, b_{r}+j-a_{j}\right)},} \\
\quad\left(r=q-p, p<q ; \Re\left(b_{r+j}\right)>\Re\left(a_{j}\right)>0 ; z \in \mathbb{C}\right),
\end{array}\right.
$$

where the generalized Beta function $B_{\gamma}^{(\alpha, \beta ; \kappa)}(x, y)$ is given by (1.3).

It is important to mention that for $\gamma=0$, equation (1.5) would reduce immediately to (1.4).

Definition 1. (Pohlen [7]) Let $f(z):=\sum_{n=0}^{\infty} a_{n} z^{n}$ and $g(z):=\sum_{n=0}^{\infty} b_{n} z^{n}$ be two power series whose radii of convergence are denoted by $R_{f}$ and $R_{g}$, respectively. Then their Hadamard product is the power series defined by

$$
(f * g)(z)=\sum_{n=0}^{\infty} a_{n} b_{n} z^{n} .
$$

The radius of convergence $R$ of the Hadamard product series $(f * g)(z)$ satisfies $R_{f} \cdot R_{g} \leq R$.

In particular, if one of the power series defines an entire function, then the Hadamard product series defines an entire function, too.

Let us consider the function ${ }_{s} F_{s+r}^{(\alpha, \beta ; \kappa, \mu)}[z ; p]$. Its decomposition is illustrative as

$$
\begin{aligned}
{ }_{s} F_{s+r}^{(\alpha, \beta ; \gamma)} & {\left[\begin{array}{c}
x_{1}, \ldots, x_{s} \\
y_{1}, \ldots, y_{s+r}
\end{array} ; z \gamma\right] } \\
& ={ }_{1} F_{r}\left[\begin{array}{c}
1, \\
y_{1}, \ldots, y_{r}
\end{array} ; z\right] *{ }_{s} F_{s}{ }^{(\alpha, \beta ; \gamma)}\left[\begin{array}{c}
x_{1}, \ldots, x_{s} \\
y_{1+r}, \ldots, y_{s+r}
\end{array} ; z ; \gamma\right] \quad(|z|<\infty) .
\end{aligned}
$$

We need to recall the following pair of the Saigo hypergeometric fractional integral operators (see Saigo [10], Kiryakova [4]). 
For $x>0, \mu, \nu, \eta \in \mathbb{C}$ and $\alpha>0$, we have

$$
\begin{aligned}
& \left(I_{0, x}^{\mu, \nu, \eta} f(t)\right)(x)=\frac{x^{-\mu-\nu}}{\Gamma(\mu)} \int_{0}^{x}(x-t)^{\mu-1}{ }_{2} F_{1}\left(\mu+\nu,-\eta ; \mu ; 1-\frac{t}{x}\right) f(t) d t, \quad(1.9) \\
& \left(J_{x, \infty}^{\mu, \nu, \eta} f(t)\right)(x)=\frac{1}{\Gamma(\mu)} \int_{x}^{\infty}(t-x)^{\mu-1} t^{-\mu-\nu}{ }_{2} F_{1}\left(\mu+\nu,-\eta ; \mu ; 1-\frac{t}{x}\right) f(t) d t
\end{aligned}
$$

where ${ }_{2} F_{1}(\cdot)$ is a special case of the Gauss hypergeometric function.

The operator $I_{0, x}^{\mu, \nu, \eta}(\cdot)$ contains both the Riemann-Liouville $R_{0, x}^{\mu}(\cdot)$ and the Erdélyi-Kober $E_{0, x}^{\mu, \eta}(\cdot)$ fractional integral operators as particular cases, by means of the relationships:

$$
\begin{gathered}
\left(R_{0, x}^{\mu} f(t)\right)(x)=\left(I_{0, x}^{\mu,-\mu, \eta} f(t)\right)(x)=\frac{1}{\Gamma(\mu)} \int_{0}^{x}(x-t)^{\mu-1} f(t) d t \\
\left(E_{0, x}^{\mu, \eta} f(t)\right)(x)=\left(I_{0, x}^{\mu, 0, \eta} f(t)\right)(x)=\frac{x^{-\mu-\eta}}{\Gamma(\mu)} \int_{0}^{x}(x-t)^{\mu-1} t^{\eta} f(t) d t .
\end{gathered}
$$

And also, note that the operator (1.10) incorporates the Weyl type and the Erdélyi-Kober fractional operators as follows:

$$
\begin{gathered}
\left(W_{x, \infty}^{\mu} f(t)\right)(x)=\left(J_{x, \infty}^{\mu,-\mu, \eta} f(t)\right)(x)=\frac{1}{\Gamma(\mu)} \int_{x}^{\infty}(t-x)^{\mu-1} f(t) d t \\
\left(K_{x, \infty}^{\mu, \eta} f(t)\right)(x)=\left(J_{x, \infty}^{\mu, 0, \eta} f(t)\right)(x)=\frac{x^{\eta}}{\Gamma(\mu)} \int_{x}^{\infty}(t-x)^{\mu-1} t^{-\mu-\eta} f(t) d t .
\end{gathered}
$$

We also use the following image formulas which are well known facts and easy consequences of the definitions of the operators (1.9) and (1.10) (see Saigo $[10])$ :

$$
\left(I_{0, x}^{\mu, \nu, \eta} t^{\lambda-1}\right)(x)=\frac{\Gamma(\lambda) \Gamma(\lambda-\nu+\eta)}{\Gamma(\lambda-\nu) \Gamma(\lambda+\mu+\eta)} x^{\lambda-\nu-1}
$$

$(\lambda>0, \lambda-\nu+\eta>0)$

$$
\left(J_{x, \infty}^{\mu, \nu, \eta} t^{\lambda-1}\right)(x)=\frac{\Gamma(\nu-\lambda+1) \Gamma(\eta-\lambda+1)}{\Gamma(1-\lambda) \Gamma(\nu+\mu-\lambda+\eta+1)} x^{\lambda-\nu-1}
$$


$(\nu-\lambda+1>0, \eta-\lambda+1>0)$.

Let $\mu, \mu^{\prime}, \nu, \nu^{\prime}, \gamma, \in \mathbb{C}, \mathbb{C}$ being the set of complex numbers and $x>0$. Then the generalized fractional derivative operators are defined as (Saigo [10])

$$
\begin{gathered}
\left(D_{0+}^{\mu, \nu, \eta} f\right)(x)=\left(I^{-\mu,-\nu, \mu+\eta} f\right)(x)=\left(\frac{d}{d x}\right)^{n}\left(I_{0+}^{-\mu+\eta,-\nu-\eta, \mu+\eta-n} f\right)(x),(1.17) \\
(\mathbb{R}(\mu) \geq 0, n=[\mathbb{R}(\mu)]+1) . \\
\left(D_{0^{-}}^{\mu, \nu, \eta} f\right)(x)=\left(I_{-}^{-\mu,-\nu, \mu+\eta} f\right)(x)=\left(-\frac{d}{d x}\right)^{n}\left(I_{-}^{-\mu+\eta,-\nu-\eta, \mu+\eta-n} f\right)(x),
\end{gathered}
$$

$$
(\mathbb{R}(\mu) \geq 0, n=[\mathbb{R}(\mu)]+1) .
$$

The operator $\left(D_{0+}^{\mu, \nu, \eta}\right)($.$) contains the Riemann-Liouville D_{0+}^{\mu}($.$) and Weyl frac-$ tional derivatives by means of the following relationships:

$$
\begin{aligned}
\left(D_{0+}^{\mu,-\mu, \eta} f\right)(x)=\left(D_{0+}^{\mu} f\right)(x)= & \left(\frac{d}{d x}\right)^{n} \frac{1}{\Gamma(n-\mu)} \int_{0}^{x} \frac{f(t) d t}{(x-t)^{\mu-n+1}}, \\
& (x>0, n=[\mathbb{R}(\mu)]+1, \mu \in \mathbb{C}, \mathbb{R}(\mu) \geq 0) .
\end{aligned}
$$

and

$$
\begin{aligned}
\left(D_{0^{-}}^{\mu,-\mu, \eta} f\right)(x)=\left(D_{-}^{\mu} f\right)(x)=\left(-\frac{d}{d x}\right)^{n} \frac{1}{\Gamma(n-\mu)} \int_{x}^{\infty} \frac{f(t) d t}{(t-x)^{\mu-n+1}}, \\
(x>0, n=[\mathbb{R}(\mu)]+1, \mu \in \mathbb{C}, \mathbb{R}(\mu) \geq 0) .
\end{aligned}
$$

It is noted that the operators (1.17), (1.18) include also the Erdélyi-Kober fractional derivative operators (Kiryakova [3]) for $\nu=0$ and $\mu, \eta \in \mathbb{C}, \mathbb{R}(\mu) \geq 0$ :

$$
\begin{aligned}
&\left(D_{0+}^{\mu, 0, \eta} f\right)(x)=\left(D_{\eta, \mu}^{+} f\right)(x)=\left(\frac{d}{d x}\right)^{n}\left(I_{0+}^{-\mu+n,-\mu,-\mu+\eta-n} f\right)(x), \\
&(x>0, n=[\mathbb{R}(\mu)]+1, \mu \in \mathbb{C}) . \\
&\left(D_{0^{-}}^{\mu, 0, \eta} f\right)(x)=\left(D_{\eta, \mu}^{-} f\right)(x)=\left(-\frac{d}{d x}\right)^{n}\left(I_{-}^{-\mu+n,-\mu,-\mu+\eta-n} f\right)(x), \\
&(x>0, n=[\mathbb{R}(\mu)]+1, \mu \in \mathbb{C}) .
\end{aligned}
$$

We also use the following image formulae which are easy consequences of the operators' definitions (Saigo [10]). Namely, for $\mu, \nu, \eta \in \mathbb{C}$ and $\mathfrak{R}(\mu) \geq 0$, $x>0, \lambda>-\min [0, \mu+\nu+\eta]$,

$$
D_{0+}^{\mu, \nu, \eta}\left(x^{\lambda-1}\right)=\frac{\Gamma(\lambda) \Gamma(\lambda+\mu+\nu+\eta)}{\Gamma(\lambda+\nu) \Gamma(\lambda+\eta)} x^{\lambda+\nu-1},
$$


and for $\mu, \nu, \eta \in \mathbb{C}$ and $x>0, \mathfrak{R}(\mu) \geq 0, \lambda<1+\min [(-\nu-n),(\mu+\eta)]$,

$$
D_{0^{-}}^{\mu, \nu, \eta}\left(x^{\lambda-1}\right)=\frac{\Gamma(1-\lambda-\nu)(1-\lambda+\mu+\eta)}{\Gamma(1-\lambda) \Gamma(1-\lambda+\eta-\nu)} x^{\lambda+\nu-1} .
$$

\section{Fractional Derivatives of Hypergeometric Function}

The right-sided Saigo fractional differentiation of the generalized Gauss hyper-

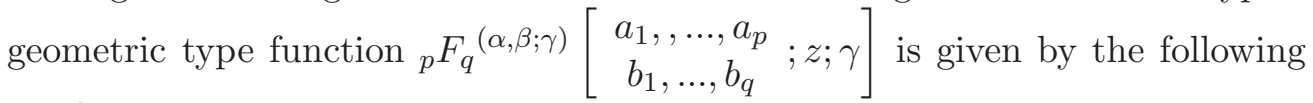
result.

Theorem 1. Let $x>0, \mathfrak{R}(\gamma)>0, \mu, \nu, \eta, \rho, e \in \mathbb{C}$ be parameters such that

$$
\mathfrak{R}(\mu)>0, \mathfrak{R}(\rho)>0, \mathfrak{R}(\rho)>-\min \{0, \mathfrak{R}(\mu+\nu+\eta)\},
$$

Then, the following fractional derivative formula holds:

$$
\begin{aligned}
D_{0+}^{\mu, \nu, \eta} & \left\{t^{\rho-1}{ }_{p} F_{q}{ }^{(\alpha, \beta ; \gamma)}\left[\begin{array}{c}
a_{1}, \ldots, a_{p} \\
b_{1}, \ldots, b_{q}
\end{array} ; e t ; \gamma\right]\right\}=x^{\rho+\nu-1} \frac{\Gamma(\rho) \Gamma(\rho+\nu+\eta)}{\Gamma(\rho+\nu) \Gamma(\rho+\eta)} \\
& \times\left\{{ }_{p} F_{q}{ }^{(\alpha, \beta ; \gamma)}\left[\begin{array}{c}
a_{1}, \ldots, a_{p} \\
b_{1}, \ldots, b_{q}
\end{array} ; e x ; \gamma\right] *{ }_{2} F_{2}\left[\begin{array}{l}
(\rho),(\rho+\nu+\eta) \\
(\rho+\nu),(\rho+\eta)
\end{array} ; e x\right]\right\} .
\end{aligned}
$$

Proof. For convenience, we denote the left-hand side of the result (2.1) by $\varsigma$. Using (1.5) and then changing the order of integration and summation, which is valid under the conditions of Theorem 1 , we find

$$
\begin{aligned}
\varsigma & =\left(D_{0+}^{\mu, \nu, \eta}\left[t^{\rho-1} \sum_{n=0}^{\infty} \Theta(n / p, q) \frac{(e t)^{n}}{n !}\right]\right) \\
& =\sum_{n=0}^{\infty} \Theta(n / p, q) \frac{e^{n}}{n !}\left[D_{0+}^{\mu, \nu, \eta}\left(t^{\rho+n-1}\right)\right] .
\end{aligned}
$$

Now, making use of the result (1.23), we obtain

$$
\varsigma=\sum_{n=0}^{\infty} \Theta(n / p, q) \frac{(e)^{n}}{n !} \frac{\Gamma(\rho+n) \Gamma(\rho+n+\nu+\eta)}{\Gamma(\rho+n+\nu) \Gamma(\rho+n+\eta)} x^{\rho+n+\nu-1}
$$

which, by applying the Hadamard product series and using equation (1.7) yields the desired result, equation (2.1). 
The left-sided Saigo fractional differentiation of the generalized Gauss hypergeometric type function ${ }_{p} F_{q}(\alpha, \beta ; \gamma)\left[\begin{array}{c}a_{1},, \ldots, a_{p} \\ b_{1}, \ldots, b_{q}\end{array} ; ; \gamma\right]$ is given by the following result.

Theorem 2. Let $x>0, \mathfrak{R}(\gamma)>0, \mu, \nu, \eta, \rho, e \in \mathbb{C}$ be parameters such that

$$
\mathfrak{R}(\mu)>0, \mathfrak{R}(\rho)<1+\min [\mathfrak{R}(-\beta-n), \mathfrak{R}(\mu+\eta)], n=\mathfrak{R}[\mu]+1 .
$$

Then, the following fractional derivative formula holds:

$$
\begin{aligned}
& D_{0-}^{\mu, \nu, \eta}\left\{t^{\rho-1}{ }_{p} F_{q}{ }^{(\alpha, \beta ; \gamma)}\left[\begin{array}{c}
a_{1},, \ldots, a_{p} \\
b_{1}, \ldots, b_{q}
\end{array} ; \frac{e}{t} ; \gamma\right]\right\} \\
& =x^{\rho-\nu-1} \frac{\Gamma(1-\rho-\nu) \Gamma(1-\rho+\mu+\eta)}{\Gamma(1-\rho) \Gamma(1-\rho-\nu+\eta)} \\
& \times\left\{{ }_{p} F_{q}^{(\alpha, \beta ; \gamma)}\left[\begin{array}{c}
a_{1}, \ldots, a_{p} \\
b_{1}, \ldots, b_{q}
\end{array} ; \frac{e}{x} ; \gamma\right] *{ }_{2} F_{2}\left[\begin{array}{c}
(1-\rho-\nu),(1-\rho+\mu+\eta) \\
(1-\rho),(1-\rho-\nu+\eta)
\end{array} ; \frac{e}{x}\right]\right\} .
\end{aligned}
$$

Proof. As in the proof of Theorem 2, taking the operator (1.18) and the result (1.24) into account, one can easily prove (2.3). Therefore, we omit the details of the proof.

Setting $\nu=0$ in Theorems 1 and 2 yield the results asserted by the following corollaries.

Corollary 1. Let $x>0, \mathfrak{R}(p) \geq 0$ and $\mu, \nu, \eta, \rho, e \in \mathbb{C}$ be parameters such that

$$
\mathfrak{R}(\mu)>0, \mathfrak{R}(\rho)>0, \mathfrak{R}(\rho)>\mathfrak{R}(-\eta) .
$$

Then the right-hand side Erdélyi-Kober fractional derivative of the generalized Gauss hypergeometric type functions is given by

$$
\begin{aligned}
& D_{0^{+}}^{\mu, 0, \eta}\left\{t^{\rho-1} F_{q}(\alpha, \beta ; \gamma)\left[\begin{array}{c}
a_{1},, \ldots, a_{p} \\
b_{1}, \ldots, b_{q}
\end{array} ; e t ; \gamma\right]\right\}= \\
& x^{\rho-1} \frac{\Gamma(\rho+\mu+\eta)}{\Gamma(\rho+\eta)}\left\{{ }_{p} F_{q}{ }^{(\alpha, \beta ; \gamma)}\left[\begin{array}{c}
a_{1},, \ldots, a_{p} \\
b_{1}, \ldots, b_{q}
\end{array} ; e x ; \gamma\right] *{ }_{1} F_{1}\left[\begin{array}{c}
(\rho+\mu+\eta) \\
(\rho+\eta)
\end{array} e x\right]\right\} \text {. }
\end{aligned}
$$


Corollary 2. Let $x>0, \mathfrak{R}(p) \geq 0$ and $\mu, \nu, \eta, \rho, e \in \mathbb{C}$ be parameters such that

$$
\mathfrak{R}(\mu)>0, \mathfrak{R}(\rho)>0, \mathfrak{R}(\rho)<1+\mathfrak{R}(\eta) .
$$

Then the left-hand side of Erdélyi-Kober fractional derivative of the generalized Gauss hypergeometric type functions is given by

$$
\begin{aligned}
& D_{0^{-}}^{\mu, 0, \eta}\left\{t^{\rho-1}{ }_{p} F_{q}(\alpha, \beta ; \gamma)\left[\begin{array}{c}
a_{1},, \ldots, a_{p} \\
b_{1}, \ldots, b_{q}
\end{array} ; \frac{e}{t} ; \gamma\right]\right\} \\
& =x^{\rho-1} \frac{\Gamma(1-\rho+\mu+\eta)}{\Gamma(1-\rho+\eta)} \\
& \times\left\{{ }_{p} F_{q}{ }^{(\alpha, \beta ; \gamma)}\left[\begin{array}{c}
a_{1}, \ldots, a_{p} \\
b_{1}, \ldots, b_{q}
\end{array} ; \frac{e}{x} ; \gamma\right] *{ }_{1} F_{1}\left[\begin{array}{c}
(1-\rho+\mu+\eta) \\
(1-\rho+\eta)
\end{array} \frac{e}{x}\right]\right\} .
\end{aligned}
$$

Further, if we replace $\nu$ with $-\mu$ in Theorems 1 and 2 and use the relations (1.19) and (1.20), we obtain the Riemann-Liouville fractional derivative of the generalized Gauss hypergeometric type function ${ }_{p} F_{q}{ }^{(\alpha, \beta ; \gamma)}\left[\begin{array}{c}a_{1},, \ldots, a_{p} \\ b_{1}, \ldots, b_{q}\end{array} ; z ; \gamma\right]$ given by the following corollaries.

Corollary 3. Let $x>0, \Re(\gamma)>0$ and $\mu \in \mathbb{C}$ be parameters such that

$$
\mathfrak{R}(\mu)>0 \text { and } \mathfrak{R}(\rho)>0
$$

Then the right-hand side Riemann-Liouville fractional derivative of the generalized Gauss hypergeometric type function is given by

$$
\begin{aligned}
& D_{0+}^{\mu,-\mu, \eta}\left\{t^{\rho-1}{ }_{p} F_{q}^{(\alpha, \beta ; \gamma)}\left[\begin{array}{c}
a_{1},, \ldots, a_{p} \\
b_{1}, \ldots, b_{q}
\end{array} ; e t ; \gamma\right]\right\} \\
& =x^{\rho-\mu-1} \frac{\Gamma(\rho) \Gamma(\rho+\eta)}{\Gamma(\rho-\mu) \Gamma(\rho+\eta)} \\
& \times\left\{{ }_{p} F_{q}{ }^{(\alpha, \beta ; \gamma)}\left[\begin{array}{c}
a_{1},, \ldots, a_{p} \\
b_{1}, \ldots, b_{q}
\end{array} ; e x ; \gamma\right] *{ }_{2} F_{2}\left[\begin{array}{c}
(\rho),(\rho+\eta) \\
(\rho-\mu),(\rho+\eta)
\end{array} ; e x\right]\right\} .
\end{aligned}
$$

Corollary 4. Let $x>0, \mathfrak{R}(\gamma)>0$ and $\mu \in \mathbb{C}$ be parameters such that

$$
\mathfrak{R}(\mu)>0 \text { and } \mathfrak{R}(\rho)>0
$$

Then the left-hand side Riemann-Liouville fractional derivative of the general- 
ized Gauss hypergeometric type function is given by

$$
\begin{aligned}
& D_{0^{-}}^{\mu,-\mu, \eta}\left[t^{\rho-1}{ }_{p} F_{q}(\alpha, \beta ; \gamma)\left[\begin{array}{c}
a_{1},, \ldots, a_{p} \\
b_{1}, \ldots, b_{q}
\end{array} ; \frac{e}{t} ; \gamma\right\}\right] \\
& =x^{\rho+\mu-1} \frac{\Gamma(1-\rho+\mu)}{\Gamma(1-\rho)} \\
& \times\left\{{ }_{p} F_{q}{ }^{(\alpha, \beta ; \gamma)}\left[\begin{array}{c}
a_{1},, \ldots, a_{p} \\
b_{1}, \ldots, b_{q}
\end{array} ; \frac{e}{x} ; \gamma\right] *{ }_{1} F_{1}\left[\begin{array}{c}
(1-\rho+\mu), \\
(1-\rho),
\end{array} ; \frac{e}{x}\right]\right\} .
\end{aligned}
$$

\section{Fractional Integral Formulas involving Generalized Hypergeometric Function}

Now the Saigo fractional integrations of generalized hypergeometric type functions are given by the following results.

Theorem 3. Let $x>0, \mathfrak{R}(\gamma)>0, \mu, \nu, \eta, \rho, e \in \mathbb{C}$ be parameters such that

$$
\mathfrak{R}(\mu)>0, \mathfrak{R}(\rho)>\max [0, \mathfrak{R}(-\nu-n)] .
$$

Then, the following fractional integral formula holds:

$$
\begin{aligned}
I_{0, x}^{\mu, \nu, \eta} & \left\{t_{p}^{\rho-1} F_{q}{ }^{(\alpha, \beta ; \gamma)}\left[\begin{array}{c}
a_{1},, \ldots, a_{p} \\
b_{1}, \ldots, b_{q}
\end{array} ; e t ; \gamma\right]\right\}(x)=x^{\rho-\nu-1} \frac{\Gamma(\rho) \Gamma(\rho+\nu+\eta)}{\Gamma(\rho-\nu) \Gamma(\rho-\mu+\eta)} \\
& \times\left\{{ }_{p} F_{q}{ }^{(\alpha, \beta ; \gamma)}\left[\begin{array}{c}
a_{1},, \ldots, a_{p} \\
b_{1}, \ldots, b_{q}
\end{array} ; e x ; \gamma\right] *{ }_{2} F_{2}\left[\begin{array}{c}
(\rho),(\rho-\nu+\eta) \\
(\rho-\nu),(\rho+\mu+\eta)
\end{array} ; e x\right]\right\} .
\end{aligned}
$$

Proof. Using (1.9), we obtain

$$
\begin{aligned}
& I_{0, x}^{\mu, \nu, \eta}\left\{t^{\rho-1}{ }_{p} F_{q}(\alpha, \beta ; \gamma)\left[\begin{array}{c}
a_{1}, \ldots, a_{p} \\
b_{1}, \ldots, b_{q}
\end{array} ; e t ; \gamma\right]\right\}(x) \\
& =\sum_{n=0}^{\infty} \Theta(n / p, q) \frac{e^{n}}{n !} I_{0, x}^{\mu, \nu, \eta}\left\{t^{n+\rho-1}\right\}(x) .
\end{aligned}
$$


Now, making use of (1.15), we get

$$
\begin{aligned}
& I_{0, x}^{\mu, \nu, \eta}\left\{t^{\rho-1} F_{q}(\alpha, \beta ; \gamma)\left[\begin{array}{c}
a_{1}, \ldots, a_{p} \\
b_{1}, \ldots, b_{q}
\end{array} ; e t ; \gamma\right]\right\}(x) \\
& =\sum_{n=0}^{\infty} \Theta(n / p, q) \frac{e^{n}}{n !} \frac{\Gamma(n+\rho) \Gamma(\rho-\nu+\eta+n)}{\Gamma(n+\rho-\nu) \Gamma(n+\rho+\mu+\eta)} x^{n+\rho-\nu-1} .
\end{aligned}
$$

The Hadamard product series and (1.5), give the desired result (3.1).

Theorem 4. Let $x>0, \mathfrak{R}(\gamma)>0, \mu, \nu, \eta, \rho, e \in \mathbb{C}$ be parameters such that

$$
\mathfrak{R}(\mu)>0, \mathfrak{R}(\rho)>\max [0, \mathfrak{R}(-\nu-\eta)] .
$$

Then, the following fractional integral formula holds:

$$
\begin{aligned}
J_{x, \infty}^{\mu, \nu, \eta} & \left\{t^{\rho-1}{ }_{p} F_{q}(\alpha, \beta ; \gamma)\left[\begin{array}{c}
a_{1}, \ldots, a_{p} \\
b_{1}, \ldots, b_{q} ; \frac{e}{t} ; \gamma
\end{array}\right]\right\}(x) \\
& =x^{\rho-\nu-1} \frac{\Gamma(\nu-\rho+1) \Gamma(1-\rho+\eta)}{\Gamma(1-\rho) \Gamma(\nu+\mu-\rho+\eta+1)} \\
& \times\left\{{ }_{p} F_{q}{ }^{(\alpha, \beta ; \gamma)}\left[\begin{array}{c}
a_{1}, \ldots, a_{p} \\
b_{1}, \ldots, b_{q} ; \frac{e}{x} ; \gamma
\end{array}\right] *{ }_{2} F_{2}\left[\begin{array}{c}
(\nu-\rho+1),(\eta-\rho+1) \\
(1-\rho),(\nu+\mu-\rho+\eta+1)
\end{array} ; \frac{e}{x}\right]\right\} .
\end{aligned}
$$

Proof. As in the proof of Theorem 3, taking the operator (1.10) and the result (1.16) into account, one can easily prove result (3.4).

Setting $\nu=0$ in Theorem 3 and Theorem 4 yield the results asserted by the following corollaries.

Corollary 5. Let $x>0, \mathfrak{R}(\gamma)>0, \mu, \nu, \eta, \rho, e \in \mathbb{C}$ be parameters such that

$$
\mathfrak{R}(\mu)>0, \mathfrak{R}(\rho)>0, \mathfrak{R}(\rho)>\mathfrak{R}(-\eta) .
$$

Then, the left-side Erdélyi-Kober fractional integral of the extended generalized hypergeometric type function is given by:

$$
\begin{aligned}
E_{0, x}^{\mu, \eta} & \left\{t^{\rho-1}{ }_{p} F_{q}{ }^{(\alpha, \beta ; \gamma)}\left[\begin{array}{c}
a_{1},, \ldots, a_{p} \\
b_{1}, \ldots, b_{q}
\end{array} ; e t ; \gamma\right]\right\}(x)=x^{\rho-1} \frac{\Gamma(\rho+\eta)}{\Gamma(\mu+\rho+\eta)} \\
& \times\left\{{ }_{p} F_{q}{ }^{(\alpha, \beta ; \gamma)}\left[\begin{array}{c}
a_{1}, \ldots, a_{p} \\
b_{1}, \ldots, b_{q}
\end{array} ; e x ; \gamma\right] *{ }_{1} F_{1}\left[\begin{array}{c}
(\rho+\eta) \\
(\rho+\eta+\mu)
\end{array} ; e x\right]\right\} .
\end{aligned}
$$


Corollary 6. Let $x>0, \mathfrak{R}(\gamma)>0, \mu, \nu, \eta, \rho, e \in \mathbb{C}$ be parameters such that

$$
\mathfrak{R}(\mu)>0, \mathfrak{R}(\rho)>0, \mathfrak{R}(\rho)<1+\mathfrak{R}(\eta) .
$$

Then, the right-side Erdélyi-Kober fractional integral of the extended generalized hypergeometric type function is given by:

$$
\begin{aligned}
K_{x, \infty}^{\mu, \eta} & \left\{t^{\rho-1}{ }_{p} F_{q}(\alpha, \beta ; \gamma)\left[\begin{array}{c}
a_{1},, \ldots, a_{p} \\
b_{1}, \ldots, b_{q}
\end{array} ; \frac{e}{t} ; \gamma\right]\right\}(x)=x^{\rho-1} \frac{\Gamma(1-\rho+\eta)}{\Gamma(1+\mu-\rho-\eta)} \\
& \times{ }_{p} F_{q}{ }^{(\alpha, \beta ; \gamma)}\left[\begin{array}{c}
a_{1}, \ldots, a_{p} \\
b_{1}, \ldots, b_{q}
\end{array} ; \frac{e}{t} ; \gamma\right] *{ }_{1} F_{1}\left[\begin{array}{c}
(1-\rho+\eta) \\
(1-\rho+\eta+\mu)
\end{array} ; \frac{e}{t}\right] .
\end{aligned}
$$

Further, if we replace $\nu$ with $-\mu$ in Theorem 3 and Theorem 4, we obtain the Riemann-Liouville fractional integrals of the generalized hypergeometric type functions given by the corollaries.

Corollary 7. Let $x>0, \mathfrak{R}(\gamma) \geq, \mu, \nu, \eta, \rho, e \in \mathbb{C}$ be parameters such that

$$
\mathfrak{R}(\mu)>0, \mathfrak{R}(\rho)>0, \mathfrak{R}(\rho)>\mathfrak{R}(-\eta) .
$$

Then, the left-side Riemann-Liouville fractional integral of the extended generalized hypergeometric type function is given by:

$$
\begin{aligned}
& R_{0, x}^{\mu}\left\{t^{\rho-1}{ }_{p} F_{q}(\alpha, \beta ; \gamma)\left[\begin{array}{c}
a_{1}, \ldots, a_{p} \\
b_{1}, \ldots, b_{q}
\end{array} ; e t ; \gamma\right]\right\}(x)=x^{\rho+\mu-1} \frac{\Gamma(\rho)}{\Gamma(\rho+\mu)} \\
& \times{ }_{p} F_{q}{ }^{(\alpha, \beta ; \gamma)}\left[\begin{array}{c}
a_{1},, \ldots, a_{p} \\
b_{1}, \ldots, b_{q}
\end{array} ; e x ; \gamma\right] *{ }_{1} F_{1}\left[\begin{array}{c}
(\rho) \\
(\rho+\mu)
\end{array} ; e x\right] .
\end{aligned}
$$

Corollary 8. Let $x>0, \mathfrak{R}(\gamma) \geq 0, \mu \in \mathbb{C}$ be parameters such that

$$
\mathfrak{R}(\mu)>0, \mathfrak{R}(\rho)>0 .
$$

Then, the right-side Riemann-Liouville fractional integral of the extended generalized hypergeometric type function is given by:

$$
\begin{aligned}
R_{x, \infty}^{\mu} & \left\{t^{\rho-1}{ }_{p} F_{q}(\alpha, \beta ; \gamma)\left[\begin{array}{c}
a_{1},, \ldots, a_{p} \\
b_{1}, \ldots, b_{q}
\end{array} ; \frac{e}{t} ; \gamma\right]\right\}(x)=x^{\rho+\mu-1} \frac{\Gamma(1-\rho-\mu)}{\Gamma(1-\rho-)} \\
& \times\left\{{ }_{p} F_{q}{ }^{(\alpha, \beta ; \gamma)}\left[\begin{array}{c}
a_{1}, \ldots, a_{p} \\
b_{1}, \ldots, b_{q}
\end{array} ; \frac{e}{t} ; \gamma\right] *{ }_{1} F_{1}\left[\begin{array}{c}
(1-\rho-\mu) \\
(1-\rho)
\end{array} ; \frac{e}{x}\right]\right\} .
\end{aligned}
$$




\section{Concluding Remarks}

The extended hypergeometric type functions defined by (1.5) have an advantage that most of the known and widely-investigated special functions are expressible in terms of the generalized Gauss hypergeometric functions. Therefore, we conclude this paper by noting that the results can lead to other numerous fractional calculus formulas for special functions, by suitable specializations of the arbitrary parameters.

\section{Acknowledgement}

Our sincere thanks are due to Krunal B. Kachhia (Department of Mathematical Sciences, Faculty of Applied Sciences, Charotar University of Science and Technology (Charusat), Changa, Anand - 388421, Gujarat, India) whose suggestions were responsible for bringing out the paper in its present form. Authors are indeed extremely grateful to the referees for their valuable suggestions for the improvement of paper.

\section{References}

[1] K.B. Kachhia and J.C. Prajapati Solution of fractional partial differential equation Aries in study of heat transfer through Diathermanous Materials, Journal of Interdisciplinary Mathematics, 18, No 1 (2015), 125-132.

[2] A.A. Kilbas, H.M. Srivastava and J.J. Trujillo, Theory and Applications of Fractinal Differential Equations, North-Holland Mathematical Studies, Elsevier Science, Amsterdem, The Netherlands (2006).

[3] V. Kiryakova, Generalized Fractional Calculus and Applications, Wiley and Sons Inc., New York (1994).

[4] V. Kiryakova, On two Saigo's fractional integral operators in the class of univalent functions, Fractional Calculus and Applied Analysis, 9, No 2 (2006), 161-176.

[5] E. Özergin, Some Properties of Hypergeometric Functions, Ph. D. Thesis, Eastern Mediterranean University, Gazimağusa, North Cyprus (2011).

[6] R.K. Parmar, A new generalization of gamma, beta, hypergeometric and confluent hypergeometric functions, Matematiche (Catania), 69 (2013), $33-52$. 
[7] T. Pohlen, The Hadamard Product and Universal Power Series, Dissertation, Universitàt Trier (2009).

[8] J.C. Prajapati and K.B. Kachhia, Fractional modeling of temperature distribution and heat flux in the semi infinite solid, Journal of Fractional Calculus and Applications, 5, No. 2 (2014), 38-43.

[9] E.D. Rainville, Special Functions, Macmillan Company, New York (1960); Reprinted by Chelsea Publishing Company, Bronx - New York (1971).

[10] M. Saigo, A remark on integral operators involving the Gauss hypergeometric functions, Math. Rep. College of General Edu. Kyushu University, 1 (1978), 135-143.

[11] S.G. Samko, A.A. Kilbas and O.I. Marichev, Fractional Integrals and Derivatives - Theory and Applications, Gordon and Breach Sci. Publ., New York (1993). 
